\title{
Short Term IPO Returns in Stock Exchange of Thailand: The Study in 2003-2013
}

\author{
Marisa Laokulrach
}

\begin{abstract}
This study examines the short term performance of initial public offering (IPO) in capital market of Thailand from 2003-2013. The daily price data is used in this study to identify the short term returns of IPO of the first trading day until the day that abnormal return cannot be earned. The results confirm the abnormal returns of the first trading day of IPO and also identify that their outperformance returns remain until day 246 after the market. IPO of four industries which are financial, service, resources, and technology outperform the market, while the other four industries which are agro and food, consumer products, industrials, and property and construction do not provide significant abnormal returns.
\end{abstract}

Index Terms-Abnormal returns, first trading day return, IPO, stock exchange of Thailand (SET).

\section{INTRODUCTION}

Issuing stocks is an importance source of funds for corporations in their expansion. A pricing process rewards the well-managed firms to have greater allocation of new investment resources.

Stock exchange of Thailand (SET), the equity market in Thailand has become an important tool for raising funds for private companies after the Asian financial crisis in 1997. Since 1997, market capitalization increased from 1,200 billion baht to about 10,000 billion baht in 2012 in which the major issuers of equities are in the eight diversified business sectors [1].

Thailand is also a part of Asian economic community (AEC) which has ASEAN Exchanges plan. ASEAN Exchange is the collaboration from Indonesia, Malaysia, Philippines, Singapore, Thailand and Vietnam. The collaboration aims to promote the growth of the ASEAN capital market by streamlining ASEAN access, introducing cross-border harmonization and creating ASEAN centric products. The ASEAN Exchanges collaboration will bring greater investment opportunities to more people as well as bring greater liquidity tocollaboration members. This collaboration aims to expand opportunities for fund raising channel [1]. SET is considered as a growing emerging capital market. In 2013, the market capitalization of initial public offerings in SET reached the highest level in ASEAN [2].

There are different studies on initial public offering (IPO) returns in both long term and short term prospect. The studies in developed markets indicate that IPO outperform the market in short term but underperform the market in long term. There are limited numbers of this study in Thailand

Manuscript received December 5, 2014; revised February 7, 2015

Marisa Laokulrach is with the Finance and Banking Department, Martin de Tours School of Management and Economics, Assumption University, Thailand (e-mail:marisalkl@au.edu). especially for short term performance of IPO.

This study is interested in measuring the first trading day returns and also short term performance of IPO stocks in Thailand's equity market. The returns of all IPO stocks in the whole market, and also of each industry are studied. While most research to date studied the monthly return up to the long term returns of IPO, this paper will examine the abnormal return of IPO over the short time horizon using daily data.

It is hypothesized that the abnormal returns of IPO are significantly higher than zero for short time horizon. This study examines the return of IPO relative to the market return to represent its abnormal return. The holding period after the listing since the first trading day up to 360 days are indicated. Then t-test statistic is used to test if the abnormal return is higher than zero significantly. The study covers 153 listing stocks from 2003-2013.

This study aims to provide useful insights of IPO performance in Thailand's equity market, and also to identify the efficiency of SET in terms of price movement aftermarket. The results will benefit both individual and institutional investor to manage their portfolio returns.

Author also expects the result to increase the numbers of listing in capital market to be the important sources of funds for private sector.

\section{STOCK EXCHANGE OF THAILAND}

From the information of the stock exchange of Thailand (SET), it has been started the operations on April 30, 1975. It operates mandatory to be a market or center for the trading of listed securities, and promoting of financial planning, as well as providing related services connected to such activities, without providing any profits to members. The main operations of SET include listing of securities, supervising of listed companies and disclosing of information, trading, and supervising members, disseminating information, and educating investor [1].

SET Index is a composite index representing the price movement for all common stocks trading on the SET. The market capitalization-weight index method is applied which the base day of April 30, 1975. SET Index is calculated from the prices of all common stocks on the main trading board. The SET also provides industry group indexes and sectoral indexes. There are eight industries in the Stock Exchange of Thailand including agro and food, consumer products, financials, industrials, resources, services, property and construction, and technology [1].

The stock market in Thailand has become an important source of funds for private firms after the Asian financial crisis in 1997. Since 1997, market capitalization increased 
from 1,200 billion baht to about 10,000 billion baht in 2012 in which the major issuers of equities are in the more diversified business sectors [1]. In 2013, there are 489 listed companies in SET. A company must apply and comply Security and Exchange Commission (SEC)'s requirements to be listed and able to trade its stocks [1], [2].

There are increasing numbers of IPO in SET after the economic crisis in 1997-2000, especially in 2003-2005 of economic expansion with 85 listing stocks. The number of IPO declined in year 2006-2011 as the results of global economic crisis. New listing stocks have increased since 2012.

In year 2013, the average daily trading value of SET surged to an all-time high and the highest level among ASEAN countries. The market capitalization of initial public offering (IPO) reached SET's record high at THB 346,506 million (USD 10,557 million), the highest level among regional stock markets. This was caused by the launch of the BTSGIF infrastructure fund of THB 62,510 million (USD 1,904 million), the largest IPO in ASEAN [2].

\section{LITERATURE REVIEW}

Numerous of international studies conclude that IPO introduce the underpricing and provide high initial returns on the first trading day. Kunz and Aggrawal [3] in 1994 found 35.8 percent average initial return in equity market in Switzerland. Ibbotson, Sindelar, and Ritter in 1994 found the 15.8 percent average return of IPO of the first trading day [4].

The studies also confirm the short term outperformance the market of IPO. IPO price performance in United State showed the significant positive buy-and-hold abnormal return from trading day 17 until day 120 , and the abnormal return reached negative on trading day 201-750 [5]. Ritter in 1991 found the 3 year holding period return of IPO was underperformed relative to the matched portfolio listed on American and New York stock exchanges [6]. Aggarwal and Rivoli in 1990 found that the short term abnormal return of IPO in New York Stock Exchange were 10.7\%, and the returns were starting to reduce after the first year [7]. IPO in Istanbul Stock Market also generated the short term abnormal return, and low long term returns. The study of IPO in Istanbul Stock Exchange by Kaya in year 2011 showed the abnormal return only at the end of third trading day [8].

The study of Alvarez, and Gonzalez in Spanish IPO revealed the non-underperformance at 12 months comparing to benchmark returns, and also identified the negative abnormal returns of $36-60$ months [9]. AbdehTabrizi and Demuri in 2003 studied the IPO in Tehran Stock Market in Iran and found the higher returns of new stocks comparing to market return if investors hold them for two months period. They also found the downward movement of accumulative abnormal return in thirty four month period [10].

Younesi, Ardekani, and Hashemijoo in 2012 studied the IPO in Malaysia and concluded that investors who are looking for new opportunities by investing in IPO, cannot gain by purchasing stocks on the listing day and hold those stocks for one year. They could gain only on the first trading day. The IPO cumulative return relative to the market show -3.77 for first week, -14.12 for a month, and -20.7 for a year after listing [11].
The IPO' abnormal returns of different industries are also studied. The study in Iranian oil and chemical industries' IPO showed the higher short term abnormal return than other industries [12]. Schaub, Casey, and Washer in 2003 found the positive abnormal returns of banking industry in New York Stock Exchange over the period 1975-1994, but less than the other non-financial industries [13].

Most existing studies of Thailand investigated the IPO in periods before 1997 economic crisis. Chorruk and Worthington in 2008 identified that IPO in SET underperformed the market at the end of month 19 aftermarket by using buy-and-hold abnormal returns method. There are limited numbers of studies on IPO' short term abnormal return in Thailand especially by using daily based data [14].

\section{DATA AND RESEARCH METHOD}

The sample of 153 new listing companies in SET during 2003-2013 is used. These IPO are from eight different industries which include 6 companies in agro and food, 3 companies in consumer products, 19 companies in financials, 19 companies in industrials, 14 companies in resources, 28 companies in services, 43 companies in property and construction, and 21 companies in technology. The issuing prices of IPO are collected from Stock Exchange of Thailand The closing price of IPO, and the closing index of SET over time horizon are collected from SET factsheets, and SETSMART (SET Market Analysis and Reporting Tool). This web-based application from SET integrates the Thai listed company data, including the historical stock prices, indexes, and listed company news.

It is hypothesized that investors can significantly earn the abnormal returns from the first trading day. Also, the abnormal returns are significantly higher than zero at 95 percent level of confidence if the investor invests in IPO stock at the offering price and hold it for short term within 1 year. Abnormal return of IPO stocks will be measured by comparing each IPO's return with the benchmark returns, which are represented by SET Index returns. The methodology of previous study by Ritter in 1991, and Casey, and Washer in 2003 are applied [6], [13].

The steps of data analysis are as follow:

First the daily return for each company $i$ for event day $t$ is calculated as:

$$
R_{i, t}=\frac{\left(P_{c-} P_{i}\right)}{P_{i}}
$$

where:

$R_{i, t}$ is the return for stock $i$ in event day $t$

$P_{i}$ is the issuing price of stock $i$

$P_{c}$ is the closing price of stock $i$ at the end of day $t$

The daily period $t$ is used up to 360 days

Second, the benchmark returns which are represented by market returns are calculated by the same method.

$$
R_{m k t, t}=\frac{\left(P_{c \_m k t-} P_{i \_m k t}\right)}{P_{i \_} m k t}
$$

where:

$R_{m k t, t}$ is the return for market in event day $t$

$P_{i \_m k t}$ is the closing index on a day before the first trading 
day

$P_{C_{-} m k t}$ is the closing index at the end of day $t$

Third, abnormal returns $A R_{i, t}$ are computed as the different of the return of stock $i$ and return of the market over the same period:

$$
A R_{i, t}=R_{i, t}-R_{m k t, t}
$$

Fourth, the average abnormal return $\overline{A R_{l, t}}$ for day $\mathrm{t}$ of $\mathrm{n}$ stocks is calculated:

$$
\overline{A R_{l, t}}=\frac{\sum_{t=1}^{n} A R_{i, t}}{n}
$$

Lastly, t-test statistic is applied to determine whether the abnormal returns are significant different from zero:

$$
A R_{-} t_{\text {day }}=\frac{\overline{A R_{l, t}}}{\sigma\left(A R_{t}\right) / \sqrt{ } n}
$$

\section{RESULTS AND CONCLUSION}

The exploring of the abnormal returns from 153 IPO stocks by using the daily data identifies the short term abnormal returns of IPO. If the investors buy the IPO stocks at the issuing price, they can significantly earn 23.83 percent average abnormal returns on the first trading day at 95 percent level of confidence.

After the first trading day, the abnormal returns decline to be at 16-20 percent until day 33 . Then they increases to 20-30 percent significantly until day 233 . From day 234 until day 246 , average abnormal returns of IPO are below 20 percent. The abnormal returns are significantly higher than zero until day 246 . The results identify the negative average abnormal returns after day 312-day 360. This confirms the short term outperformance of IPO, but long term underperformance the market of IPO in the equity market of Thailand (Table I).

\begin{tabular}{|c|c|c|c|c|}
\hline Day 24 & 19.369 & 59.538 & 3.154 & 0.001 \\
\hline Day 25 & 20.331 & 62.642 & 3.147 & 0.001 \\
\hline Day 26 & 19.798 & 61.618 & 3.115 & 0.001 \\
\hline Day 27 & 20.458 & 63.399 & 3.128 & 0.001 \\
\hline Day 28 & 20.130 & 62.639 & 3.116 & 0.001 \\
\hline Day 29 & 20.012 & 62.155 & 3.122 & 0.001 \\
\hline \multirow[t]{2}{*}{ Day 30} & 19.712 & 61.206 & 3.123 & 0.001 \\
\hline & $\begin{array}{c}\text { Average } \\
\text { AR }\end{array}$ & $\begin{array}{c}\text { Standard } \\
\text { Deviation }\end{array}$ & $t$-statistic & $p$ value \\
\hline Day 31 & 19.733 & 60.819 & 3.146 & 0.001 \\
\hline Day 32 & 20.262 & 61.771 & 3.180 & 0.001 \\
\hline Day 33 & 19.965 & 60.782 & 3.185 & 0.001 \\
\hline Day 34 & 20.384 & 61.541 & 3.211 & 0.001 \\
\hline Day 35 & 21.239 & 62.145 & 3.314 & 0.001 \\
\hline Day 36 & 22.605 & 64.852 & 3.380 & 0.001 \\
\hline Day 37 & 22.460 & 64.454 & 3.360 & 0.001 \\
\hline Day 38 & 22.960 & 65.608 & 3.393 & 0.001 \\
\hline Day 39 & 23.024 & 65.148 & 3.426 & 0.001 \\
\hline Day 40 & 23.452 & 64.967 & 3.500 & 0.001 \\
\hline Day 41 & 23.692 & 65.093 & 3.529 & 0.001 \\
\hline Day 42 & 23.975 & 65.437 & 3.552 & 0.001 \\
\hline Day 43 & 23.865 & 65.639 & 3.525 & 0.001 \\
\hline Day 44 & 24.752 & 66.724 & 3.597 & 0.001 \\
\hline Day 45 & 25.301 & 68.952 & 3.558 & 0.001 \\
\hline Day 46 & 25.180 & 69.198 & 3.528 & 0.001 \\
\hline Day 47 & 24.237 & 68.210 & 3.445 & 0.001 \\
\hline Day 48 & 24.697 & 68.305 & 3.506 & 0.001 \\
\hline Day 49 & 24.650 & 68.974 & 3.465 & 0.001 \\
\hline Day 50 & 25.947 & 67.303 & 3.718 & 0.000 \\
\hline Day 51 & 25.484 & 67.719 & 3.629 & 0.000 \\
\hline Day 52 & 25.260 & 70.282 & 3.485 & 0.001 \\
\hline Day 53 & 25.785 & 71.344 & 3.485 & 0.001 \\
\hline Day 54 & 25.736 & 71.654 & 3.482 & 0.001 \\
\hline Day 55 & 25.583 & 72.120 & 3.439 & 0.001 \\
\hline Day 56 & 26.311 & 75.816 & 3.365 & 0.001 \\
\hline Day 57 & 27.456 & 74.227 & 3.567 & 0.001 \\
\hline Day 58 & 25.984 & 74.825 & 3.367 & 0.001 \\
\hline Day 59 & 26.167 & 75.279 & 3.370 & 0.001 \\
\hline Day 60 & 26.764 & 74.641 & 3.477 & 0.001 \\
\hline Day 61 & 26.710 & 75.495 & 3.430 & 0.001 \\
\hline Day 62 & 26.210 & 74.538 & 3.409 & 0.001 \\
\hline Day 63 & 27.330 & 76.654 & 3.457 & 0.001 \\
\hline Day 64 & 27.774 & 77.689 & 3.466 & 0.001 \\
\hline Day 65 & 28.201 & 78.648 & 3.477 & 0.001 \\
\hline Day 66 & 28.444 & 77.907 & 3.540 & 0.001 \\
\hline Day 67 & 28.419 & 78.518 & 3.509 & 0.001 \\
\hline Day 68 & 28.950 & 77.973 & 3.600 & 0.001 \\
\hline Day 69 & 30.255 & 79.652 & 3.683 & 0.000 \\
\hline Day 70 & 30.124 & 79.102 & 3.692 & 0.000 \\
\hline Day 71 & 30.842 & 80.214 & 3.728 & 0.000 \\
\hline Day 72 & 30.169 & 79.623 & 3.674 & 0.000 \\
\hline Day 73 & 28.930 & 79.344 & 3.535 & 0.001 \\
\hline Day 74 & 29.428 & 78.224 & 3.647 & 0.000 \\
\hline Day 75 & 29.379 & 77.305 & 3.685 & 0.000 \\
\hline Day 76 & 29.646 & 76.571 & 3.754 & 0.000 \\
\hline Day 77 & 29.680 & 75.156 & 3.829 & 0.000 \\
\hline Day 78 & 30.138 & 75.504 & 3.870 & 0.000 \\
\hline Day 79 & 28.964 & 74.848 & 3.752 & 0.000 \\
\hline Day 80 & 29.668 & 75.949 & 3.787 & 0.000 \\
\hline Day 81 & 29.297 & 78.074 & 3.638 & 0.000 \\
\hline Day 82 & 30.970 & 77.703 & 3.864 & 0.000 \\
\hline Day 83 & 30.197 & 77.627 & 3.751 & 0.000 \\
\hline
\end{tabular}

TABLE I: ABNORMAL RETURNS OF IPO

\begin{tabular}{lcccc}
\hline & $\begin{array}{c}\text { Average } \\
\text { AR }\end{array}$ & $\begin{array}{c}\text { Standard } \\
\text { Deviation }\end{array}$ & $t$-statistic & $p$ value \\
\hline Day 1 & 23.830 & 41.306 & 5.593 & 0.000 \\
\hline Day 2 & 20.749 & 42.509 & 4.732 & 0.000 \\
\hline Day 3 & 20.271 & 44.418 & 4.425 & 0.000 \\
\hline Day 4 & 21.171 & 45.459 & 4.515 & 0.000 \\
\hline Day 5 & 19.832 & 44.340 & 4.337 & 0.000 \\
\hline Day 6 & 19.988 & 46.197 & 4.195 & 0.000 \\
\hline Day 7 & 19.892 & 47.080 & 4.096 & 0.000 \\
\hline Day 8 & 19.519 & 46.826 & 4.041 & 0.000 \\
\hline Day 9 & 18.268 & 46.364 & 3.820 & 0.000 \\
\hline Day 10 & 17.952 & 47.259 & 3.683 & 0.000 \\
\hline Day 11 & 17.875 & 46.576 & 3.721 & 0.000 \\
\hline Day 12 & 16.920 & 46.902 & 3.498 & 0.001 \\
\hline Day 13 & 17.036 & 48.386 & 3.414 & 0.001 \\
\hline Day 14 & 16.751 & 48.629 & 3.340 & 0.001 \\
\hline Day 15 & 16.767 & 48.160 & 3.375 & 0.001 \\
\hline Day 16 & 16.841 & 48.464 & 3.369 & 0.001 \\
\hline Day 17 & 17.153 & 50.190 & 3.313 & 0.001 \\
\hline Day 18 & 17.283 & 50.859 & 3.295 & 0.001 \\
\hline Day 19 & 17.843 & 51.896 & 3.334 & 0.001 \\
\hline Day 20 & 18.065 & 52.692 & 3.324 & 0.001 \\
\hline Day 21 & 18.836 & 54.852 & 3.329 & 0.001 \\
\hline Day 22 & 19.188 & 56.950 & 3.267 & 0.001 \\
\hline Day 23 & 19.701 & 60.728 & 3.145 & 0.001 \\
\hline & & & &
\end{tabular}




\begin{tabular}{|c|c|c|c|c|}
\hline Day 84 & 30.143 & 77.052 & 3.793 & 0.000 \\
\hline Day 85 & 30.064 & 75.849 & 3.843 & 0.000 \\
\hline Day 86 & 29.840 & 74.980 & 3.859 & 0.000 \\
\hline Day 87 & 29.572 & 74.397 & 3.854 & 0.000 \\
\hline Day 88 & 30.391 & 75.966 & 3.879 & 0.000 \\
\hline Day 88 & 30.391 & 75.966 & 3.879 & 0.000 \\
\hline \multirow[t]{2}{*}{ Day 89} & 29.752 & 75.419 & 3.825 & 0.000 \\
\hline & $\begin{array}{c}\text { Average } \\
\text { AR }\end{array}$ & $\begin{array}{l}\text { Standard } \\
\text { Deviation }\end{array}$ & $t$-statistic & $p$ value \\
\hline Day 90 & 30.149 & 75.800 & 3.856 & 0.000 \\
\hline Day 91 & 32.194 & 80.867 & 3.860 & 0.000 \\
\hline Day 92 & 32.306 & 79.421 & 3.944 & 0.000 \\
\hline Day 93 & 31.923 & 79.099 & 3.913 & 0.000 \\
\hline Day 94 & 31.673 & 77.031 & 3.986 & 0.000 \\
\hline Day 95 & 30.598 & 75.494 & 3.930 & 0.000 \\
\hline Day 96 & 29.769 & 74.498 & 3.874 & 0.000 \\
\hline Day 97 & 31.021 & 75.118 & 4.004 & 0.000 \\
\hline Day 98 & 31.186 & 75.584 & 3.979 & 0.000 \\
\hline Day 99 & 30.891 & 74.765 & 4.006 & 0.000 \\
\hline Day 100 & 30.574 & 74.247 & 3.992 & 0.000 \\
\hline Day 101 & 29.464 & 73.341 & 3.895 & 0.000 \\
\hline Day 102 & 29.009 & 73.435 & 3.830 & 0.000 \\
\hline Day 103 & 29.025 & 73.135 & 3.848 & 0.000 \\
\hline Day 104 & 29.738 & 76.247 & 3.781 & 0.000 \\
\hline Day 105 & 30.267 & 79.520 & 3.690 & 0.000 \\
\hline Day 106 & 30.115 & 79.147 & 3.669 & 0.000 \\
\hline Day 107 & 30.648 & 79.258 & 3.729 & 0.000 \\
\hline Day 108 & 29.288 & 79.595 & 3.549 & 0.001 \\
\hline Day 109 & 29.404 & 78.862 & 3.615 & 0.000 \\
\hline Day 110 & 29.511 & 76.406 & 3.745 & 0.000 \\
\hline Day 111 & 29.063 & 74.160 & 3.800 & 0.000 \\
\hline Day 112 & 29.710 & 76.125 & 3.764 & 0.000 \\
\hline Day 113 & 29.375 & 75.956 & 3.750 & 0.000 \\
\hline Day 114 & 29.603 & 75.193 & 3.817 & 0.000 \\
\hline Day 115 & 29.894 & 75.337 & 3.827 & 0.000 \\
\hline Day 116 & 29.361 & 75.715 & 3.760 & 0.000 \\
\hline Day 117 & 28.799 & 74.940 & 3.726 & 0.000 \\
\hline Day 118 & 28.782 & 75.162 & 3.713 & 0.000 \\
\hline Day 119 & 27.009 & 74.703 & 3.505 & 0.001 \\
\hline Day 120 & 27.925 & 74.358 & 3.641 & 0.000 \\
\hline Day 121 & 27.525 & 75.643 & 3.528 & 0.001 \\
\hline Day 122 & 27.891 & 76.766 & 3.523 & 0.001 \\
\hline Day 123 & 27.578 & 76.548 & 3.474 & 0.001 \\
\hline Day 124 & 27.712 & 78.003 & 3.426 & 0.001 \\
\hline Day 125 & 27.800 & 78.833 & 3.419 & 0.001 \\
\hline Day 126 & 27.683 & 78.198 & 3.432 & 0.001 \\
\hline Day 127 & 27.171 & 77.357 & 3.387 & 0.001 \\
\hline Day 128 & 26.491 & 76.213 & 3.370 & 0.001 \\
\hline Day 129 & 27.104 & 76.905 & 3.417 & 0.001 \\
\hline Day 130 & 27.286 & 75.714 & 3.475 & 0.001 \\
\hline Day 131 & 26.699 & 75.986 & 3.407 & 0.001 \\
\hline Day 132 & 26.977 & 75.669 & 3.456 & 0.001 \\
\hline Day 133 & 27.146 & 77.220 & 3.408 & 0.001 \\
\hline Day 134 & 26.828 & 76.479 & 3.401 & 0.001 \\
\hline Day 135 & 26.628 & 76.698 & 3.366 & 0.001 \\
\hline Day 136 & 25.401 & 77.588 & 3.174 & 0.001 \\
\hline Day 137 & 24.687 & 77.843 & 3.058 & 0.002 \\
\hline Day 138 & 23.758 & 76.721 & 3.002 & 0.002 \\
\hline Day 139 & 23.332 & 75.644 & 2.990 & 0.002 \\
\hline Day 140 & 23.310 & 74.842 & 3.020 & 0.002 \\
\hline Day 141 & 23.107 & 75.321 & 2.974 & 0.002 \\
\hline Day 142 & 23.400 & 75.884 & 2.990 & 0.002 \\
\hline
\end{tabular}

\begin{tabular}{|c|c|c|c|c|}
\hline Day 143 & 21.342 & 76.022 & 2.722 & 0.004 \\
\hline Day 144 & 20.946 & 75.259 & 2.698 & 0.004 \\
\hline Day 145 & 20.637 & 74.794 & 2.675 & 0.005 \\
\hline Day 146 & 21.316 & 76.492 & 2.702 & 0.004 \\
\hline Day 147 & 21.718 & 77.736 & 2.694 & 0.004 \\
\hline Day 148 & 22.358 & 77.937 & 2.781 & 0.004 \\
\hline \multirow[t]{2}{*}{ Day 149} & 22.518 & 79.362 & 2.736 & 0.004 \\
\hline & $\begin{array}{c}\text { Average } \\
\text { AR }\end{array}$ & $\begin{array}{c}\text { Standard } \\
\text { Deviation }\end{array}$ & $t$-statistic & $p$ value \\
\hline Day 150 & 22.714 & 79.182 & 2.781 & 0.004 \\
\hline Day 151 & 23.450 & 80.402 & 2.828 & 0.003 \\
\hline Day 152 & 22.173 & 77.411 & 2.777 & 0.004 \\
\hline Day 153 & 20.832 & 78.937 & 2.559 & 0.006 \\
\hline Day 154 & 20.919 & 78.327 & 2.589 & 0.006 \\
\hline Day 155 & 22.113 & 80.304 & 2.656 & 0.005 \\
\hline Day 156 & 23.785 & 79.233 & 2.895 & 0.003 \\
\hline Day 157 & 22.438 & 78.085 & 2.786 & 0.003 \\
\hline Day 158 & 21.876 & 78.481 & 2.703 & 0.004 \\
\hline Day 159 & 21.614 & 79.724 & 2.628 & 0.005 \\
\hline Day 160 & 22.518 & 78.593 & 2.778 & 0.004 \\
\hline Day 161 & 21.484 & 79.652 & 2.615 & 0.005 \\
\hline Day 162 & 22.997 & 76.757 & 2.889 & 0.003 \\
\hline Day 163 & 24.287 & 77.247 & 3.016 & 0.002 \\
\hline Day 164 & 23.543 & 78.407 & 2.911 & 0.003 \\
\hline Day 165 & 25.220 & 80.616 & 3.017 & 0.002 \\
\hline Day 166 & 24.706 & 81.382 & 2.928 & 0.002 \\
\hline Day 167 & 24.155 & 80.356 & 2.914 & 0.002 \\
\hline Day 168 & 22.827 & 80.719 & 2.742 & 0.004 \\
\hline Day 169 & 23.936 & 80.139 & 2.896 & 0.003 \\
\hline Day 170 & 24.036 & 82.356 & 2.830 & 0.003 \\
\hline Day 171 & 23.829 & 81.780 & 2.825 & 0.003 \\
\hline Day 172 & 23.266 & 82.645 & 2.729 & 0.004 \\
\hline Day 173 & 22.166 & 81.599 & 2.634 & 0.005 \\
\hline Day 174 & 24.097 & 80.537 & 2.901 & 0.003 \\
\hline Day 175 & 24.678 & 80.621 & 2.952 & 0.002 \\
\hline Day 176 & 23.901 & 82.366 & 2.813 & 0.003 \\
\hline Day 177 & 24.895 & 82.138 & 2.939 & 0.002 \\
\hline Day 178 & 24.987 & 82.760 & 2.896 & 0.003 \\
\hline Day 179 & 24.026 & 81.907 & 2.844 & 0.003 \\
\hline Day 180 & 24.246 & 81.652 & 2.864 & 0.003 \\
\hline Day 181 & 23.754 & 81.199 & 2.836 & 0.003 \\
\hline Day 182 & 22.709 & 79.758 & 2.761 & 0.004 \\
\hline Day 183 & 22.959 & 82.016 & 2.700 & 0.004 \\
\hline Day 184 & 23.491 & 81.737 & 2.772 & 0.004 \\
\hline Day 185 & 23.511 & 81.995 & 2.780 & 0.004 \\
\hline Day 186 & 24.085 & 83.218 & 2.806 & 0.003 \\
\hline Day 187 & 25.203 & 85.253 & 2.851 & 0.003 \\
\hline Day 188 & 24.742 & 84.173 & 2.850 & 0.003 \\
\hline Day 189 & 24.346 & 84.294 & 2.800 & 0.003 \\
\hline Day 190 & 21.887 & 83.263 & 2.549 & 0.006 \\
\hline Day 191 & 24.418 & 84.380 & 2.806 & 0.003 \\
\hline Day 192 & 24.306 & 84.761 & 2.780 & 0.004 \\
\hline Day 193 & 23.758 & 85.273 & 2.701 & 0.004 \\
\hline Day 194 & 23.996 & 86.270 & 2.697 & 0.004 \\
\hline Day 195 & 23.816 & 86.349 & 2.674 & 0.005 \\
\hline Day 196 & 23.753 & 86.477 & 2.663 & 0.005 \\
\hline Day 197 & 24.487 & 88.795 & 2.674 & 0.005 \\
\hline Day 198 & 26.119 & 90.641 & 2.779 & 0.004 \\
\hline Day 199 & 24.515 & 89.041 & 2.669 & 0.005 \\
\hline Day 200 & 26.025 & 91.050 & 2.757 & 0.004 \\
\hline Day 201 & 22.855 & 90.563 & 2.447 & 0.008 \\
\hline Day 202 & 24.081 & 89.166 & 2.618 & 0.005 \\
\hline
\end{tabular}




\begin{tabular}{|c|c|c|c|c|}
\hline Day 203 & 24.907 & 90.253 & 2.676 & 0.005 \\
\hline Day 204 & 22.705 & 91.070 & 2.417 & 0.009 \\
\hline Day 205 & 24.769 & 88.836 & 2.703 & 0.004 \\
\hline Day 206 & 24.176 & 88.286 & 2.655 & 0.005 \\
\hline Day 207 & 25.112 & 88.069 & 2.750 & 0.004 \\
\hline Day 208 & 24.426 & 88.064 & 2.689 & 0.004 \\
\hline \multirow[t]{2}{*}{ Day 209} & 23.848 & 87.795 & 2.620 & 0.005 \\
\hline & $\begin{array}{c}\text { Average } \\
\text { AR }\end{array}$ & $\begin{array}{l}\text { Standard } \\
\text { Deviation }\end{array}$ & $t$-statistic & $p$ value \\
\hline Day 210 & 23.931 & 85.604 & 2.696 & 0.004 \\
\hline Day 211 & 23.373 & 85.491 & 2.637 & 0.005 \\
\hline Day 212 & 22.954 & 88.847 & 2.491 & 0.008 \\
\hline Day 213 & 24.251 & 88.105 & 2.654 & 0.005 \\
\hline Day 214 & 23.825 & 88.051 & 2.595 & 0.006 \\
\hline Day 215 & 24.078 & 88.107 & 2.621 & 0.005 \\
\hline Day 216 & 24.409 & 88.239 & 2.668 & 0.005 \\
\hline Day 217 & 23.073 & 88.911 & 2.503 & 0.007 \\
\hline Day 218 & 24.343 & 88.608 & 2.649 & 0.005 \\
\hline Day 219 & 23.445 & 88.100 & 2.553 & 0.006 \\
\hline Day 220 & 21.373 & 87.440 & 2.345 & 0.011 \\
\hline Day 221 & 22.548 & 85.962 & 2.516 & 0.007 \\
\hline Day 222 & 22.028 & 85.592 & 2.468 & 0.008 \\
\hline Day 223 & 21.860 & 84.258 & 2.488 & 0.008 \\
\hline Day 224 & 23.635 & 85.699 & 2.616 & 0.005 \\
\hline Day 225 & 21.182 & 84.267 & 2.411 & 0.009 \\
\hline Day 226 & 21.809 & 84.018 & 2.476 & 0.008 \\
\hline Day 227 & 20.534 & 85.156 & 2.313 & 0.012 \\
\hline Day 228 & 20.794 & 84.569 & 2.358 & 0.010 \\
\hline Day 229 & 19.479 & 84.403 & 2.202 & 0.015 \\
\hline Day 230 & 20.969 & 82.751 & 2.430 & 0.009 \\
\hline Day 231 & 20.876 & 82.825 & 2.418 & 0.009 \\
\hline Day 232 & 21.379 & 82.904 & 2.460 & 0.008 \\
\hline Day 233 & 21.200 & 82.015 & 2.452 & 0.008 \\
\hline Day 234 & 19.665 & 81.711 & 2.296 & 0.012 \\
\hline Day 235 & 19.500 & 79.140 & 2.363 & 0.010 \\
\hline Day 236 & 19.446 & 81.198 & 2.285 & 0.013 \\
\hline Day 237 & 15.201 & 78.981 & 1.846 & 0.034 \\
\hline Day 238 & 14.997 & 77.827 & 1.848 & 0.034 \\
\hline Day 239 & 14.075 & 75.711 & 1.783 & 0.039 \\
\hline Day 240 & 14.675 & 76.578 & 1.838 & 0.035 \\
\hline Day 241 & 14.132 & 77.650 & 1.746 & 0.042 \\
\hline Day 242 & 14.139 & 77.579 & 1.748 & 0.042 \\
\hline Day 243 & 13.809 & 77.639 & 1.706 & 0.046 \\
\hline Day 244 & 14.034 & 77.657 & 1.733 & 0.043 \\
\hline Day 245 & 13.804 & 77.547 & 1.707 & 0.046 \\
\hline Day 246 & 13.896 & 77.762 & 1.714 & 0.045 \\
\hline
\end{tabular}

Investors can earn high abnormal returns at 60-70 percent on day 191 to day 233 , then the performance of this industry's IPO decrease to be at 50-60 percent on trading day 234 to day 246.

TABLE II: ABNORMAL RETURNS OF IPO FROM DIFFERENT INDUSTRIES

\begin{tabular}{lcccc}
\hline \hline Industry & $\begin{array}{c}\text { First Trading } \\
\text { Day Abnormal } \\
\text { Return }\end{array}$ & $t$-statistic & $p$-value & $\begin{array}{c}\text { Periods of } \\
\text { Abnormal } \\
\text { Return }\end{array}$ \\
\hline Financial & $45.93 \%$ & 2.470 & 0.150 & $\begin{array}{c}\text { until } 246 \\
\text { days }\end{array}$ \\
\hline Service & $34.88 \%$ & 3.065 & 0.004 & $\begin{array}{c}\text { until 137 } \\
\text { days }\end{array}$ \\
\hline Resources & $30.53 \%$ & 3.343 & 0.005 & $\begin{array}{c}\text { until 120 } \\
\text { days }\end{array}$ \\
\hline Technology & $28.47 \%$ & 2.748 & 0.007 & $\begin{array}{c}\text { until } 125 \\
\text { days }\end{array}$ \\
\hline
\end{tabular}

Service industry provides 34.88 percent abnormal returns on the first trading day with the period of 137 days aftermarket of generating abnormal returns. The abnormal returns from trading day 2 to day 32 are at 23 to 35 percent. Trading day 33 to day 79 provide the abnormal returns of 35 to 50 percent, then they increases to be at 45 to 55 percent during day 80 to day 137 .

Resources industry' IPO stocks provide the 30.53 percent abnormal returns on the first trading day with 120 days of earning the abnormal returns. The average abnormal returns from the second trading day to day 120 are at 25 to 40 percent.

IPO of technology industry can generate 28.47 percent abnormal returns on the first trading day with the 125 trading days of outperformance. The average abnormal returns are at 20 to 35 percent.

The other four industries which are agro and food, consumer products, industrials, and property and construction do not significantly provide abnormal returns on the first trading day and also other days after listing. The results show the negative average returns of IPO in consumption industry on the first trading day. IPO in property industry provide significantly negative abnormal returns on 293 days aftermarket.

This study's results identify the inefficiency of pricing movement of IPO in capital market in Thailand, which provides the opportunity for investors to generate abnormal returns from this event. The analysis of daily abnormal returns after offerings also contributes the different viewpoint of short term performance of IPO from other studies.

The research's results benefit both individual and also

The study of IPO in different 8 industries was also examined. Table II shows that four industries which are financial, service, resources, and technology provide significant positive abnormal returns at 95 percent level of confidence.IPO of financial industry provides the highest first trading day abnormal returns of 45.93 percent followed by service, resources, and technology industries (Table II).

Financial industry provides the longest period of 246 trading days aftermarket to earn the abnormal return. The results show that financial industry's IPO provide higher abnormal returns than other industries. From day 2 to day 22, the abnormal returns are at 35-45 percent. Then abnormal returns increases to be between 45-75 percent from trading day 23 until day 139. Then the abnormal returns are at the level of 50-60 percent during trading day 140 to day 190 . institutional investors such as mutual funds management companies to manage their portfolios by including the IPO stocks and hold them for short term period to outperform the market. The four industries should be emphasized in generating abnormal returns. The insight information in term of number of days to hold the IPO in a portfolio to generate significant positive abnormal returns is also useful for the investors.

The author highly expects that the outperforming of IPO over the market return can encourage the investors to invest more in equity market. This finally provides more opportunities for private corporations to raise funds by issuing stocks for their expansion and to promote the growth and stabilize of economy and Stock Exchange of Thailand, especially as the leading equity market in ASEAN. 


\section{REFERENCES}

[1] SET Annual Report, Stock Exchange of Thailand, Bangkok, pp. 10-20, 2012.

[2] SET Annual Report, Stock Exchange of Thailand, Bangkok, pp. 10-25, 2013.

[3] R. M. Kunz and R. A. Reena, "Why initial public offerings are underpriced: Evidence from Switzerland," Journal of Banking and Finance, vol. 18, no. 4, pp. 705-723, 1994.

[4] R. G. Ibbotson, J. L. Sindelar, and J. R. Ritter, "The market's problems with the pricing of initial public offerings," Journal of Applied Corporate Finance, vol. 7, pp. 66-74, 1994.

[5] Z. Smith, "An empirical analysis of initial public offering (IPO) price performance in Unitied States," Journal of Finance and Accountancy, An Empirical Analysis, pp. 1-19, 2009.

[6] J. R. Ritter, "The long-run performance of initial public offerings," Journal of Finance, vol. 1, pp. 3-27, 1991.

[7] R. Aggarwal and P. Rivoli, "Fads in the initial public offering market?" The Journal of the Financial Management Association, vol. 19, no. 4, pp. 45-57, 1990.

[8] T. Kaya, "The short term performance of initial public offerings in Istanbul stock exchange: 2010-2011 application," Journal of Business, Economics \& Finance, vol. 1, no. 1, pp. 64-76, 2012.

[9] S. Alvarez and V. Gonzalez. (2011). Long-run performance of initial public offerings (IPO) in Spanish capital market. [Online]. Available: http://dx.doi.org/10.2139/ssrn.274086

[10] T. H. AbdehTabrizi and D. Dariush, "Identifying the effective factors in stock return of newly accepted firms in tehran stock market," Financial Researches Quarterly Periodical, vol. 5, no. 15, pp. 24-30, 2003.
[11] N. Younesi, A. M. Ardekani, and M. Hashemijoo, "Performance of Malaysian IPO and impact of return determinants," Journal of Business Studies Quarterly, vol. 4, no. 2, pp. 140-158, 2012.

[12] M. Filsaraei, A. Azarberahman, and J. Azarberahman, "An empirical analysis for abnormal returns from initial public offerings (IPO): Evidence of Iranian oil and chemical industries," International Journal of Accounting and Financial Reporting, vol. 3, no. 1, pp. 143-161, 2013.

[13] M. Schaub, M. K. Casey, and K. M. Washer, "Banking industry IPO returns: A test of the informational asymmetry hypothesis," Southwest Business and Economics Journal, vol. 12, pp. 19-24, 2003-2004.

[14] J. Chorruk and A. C. Worthington, "New evidence on the pricing performance of initial public offerings in Thailand," Emerging Markets Review, vol. 11, no. 3, pp. 285-299, 2010.

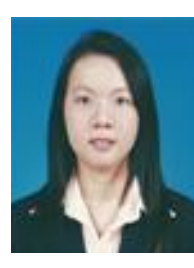

Marisa Laokulrach was born in 1976 in Bangkok, Thailand. She graduated with a Ph.D. degree in development administration (international) from The National Institute of Development Administration (NIDA), Thailand. She received the master degree in business administration (MBA) and the bachelor degree in business administration, majoring in finance and banking from Assumption University (ABAC), Thailand. Currently, she is a full time lecturer in the Department of Finance and Banking, Martin de Tours School of Management and Economics, Assumption University (ABAC), Thailand. She is interested in the research areas on equity market, investment, and public finance. 